\title{
Head-mounted accelerometry accurately detects prey capture in California sea lions
}

\author{
Mason R. Cole* ${ }^{*}$, Jenifer A. Zeligs, Stefani Skrovan and Birgitte I. McDonald
}

\begin{abstract}
Detecting when and where animals feed is key to understanding their ecophysiology, but our ability to collect these data in marine mammals remains limited. Here, we test a tag-based accelerometry method to detect prey capture in California sea lions. From synchronized underwater video and acceleration data of two trained sea lions, we isolated a combined acceleration and Jerk pattern that reliably indicated prey capture in training datasets. We observed a stereotyped feeding motion in underwater video that included (1) mouth opening while approaching prey; (2) head deceleration to allow initial suction or prey engulfment, and (3) jaw closure. This motion (1-3) was repeated if a prey item was not initially engulfed. This stereotyped feeding motion informed a signal pattern phrase that accurately detected feeding in a training dataset. This phrase required (1) an initial heave-axis Jerk signal surpassing a threshold based on sampling rate; (2) an estimated dynamic surge-axis deceleration signal surpassing $-0.7 \mathrm{~g}$ beginning within $0.2 \mathrm{~s}$ of the initial Jerk signal; and (3) an estimated dynamic surge-axis acceleration signal surpassing $1.0 \mathrm{~g}$ within $0.5 \mathrm{~s}$ of the beginning of the prior deceleration signal. We built an automated detector in MATLAB to identify and quantify these patterns. Blind tests of this detector on non-training datasets found high true-positive detection rates (91\%100\%) with acceleration sampled at $50-333 \mathrm{~Hz}$ and low false-positive detection rates (0\%-4.8\%) at all sampling rates $(16-333 \mathrm{~Hz})$. At $32 \mathrm{~Hz}$ and below, true-positive detection rates decreased due to attenuation of signal detail. A detector optimized for an adult female was also accurate at 32-100 Hz when tested on an adult male's data, suggesting the potential future use of a generalized detector in wild subjects. When tested on the same data, a published triaxial Jerk method produced high true-positive detection rates (91-100\%) and low-to-moderate false-positive detection rates $(15-43 \%)$ at $\geq 32 \mathrm{~Hz}$. Using our detector, larger prey elicited longer prey capture duration in both animals at almost all sampling rates $32 \mathrm{~Hz}$ or faster. We conclude that this method can accurately detect feeding and estimate relative prey length in California sea lions.
\end{abstract}

Keywords: California sea lion, Accelerometry, Feeding, Automated detection, Biologging, Prey capture, Signal analysis, Otariid

\section{Background}

Quantitative feeding observations are key in determining an animal's foraging efficiency, ecophysiology, and ecological impact (e.g., $[5,8,39])$, but our ability to collect these data in many marine mammals remains limited. Marine mammal foraging behavior has for decades been

*Correspondence: mcole@mlml.calstate.edu

Moss Landing Marine Labs, San Jose State University, 8272 Moss Landing Rd., Moss Landing, CA 95039, USA assumed from depth profiles (e.g., 'Wiggles') and movement patterns (e.g., area-restricted search) during dives and foraging trips $[3,7,16,18]$. While useful to infer behavioral state, these methods cannot resolve individual feeding attempts in most species, and must be groundtruthed to produce reliable quantitative feeding data $[29,34,36]$. Animal-borne video cameras can directly observe feeding and estimate prey size and species [2, $4,26]$, but are limited by restrictive battery life and may potentially bias results if a light source is used at depth. Furthermore, high costs and extensive video analysis original author(s) and the source, provide a link to the Creative Commons licence, and indicate if changes were made. The images or other third party material in this article are included in the article's Creative Commons licence, unless indicated otherwise in a credit line to the material. If material is not included in the article's Creative Commons licence and your intended use is not permitted by statutory regulation or exceeds the permitted use, you will need to obtain permission directly from the copyright holder. To view a copy of this licence, visit http://creativecommons.org/licenses/by/4.0/. The Creative Commons Public Domain Dedication waiver (http://creativeco mmons.org/publicdomain/zero/1.0/) applies to the data made available in this article, unless otherwise stated in a credit line to the data. 
following collection will limit the extent of deployments and may render the use of video cameras impractical or unviable for many studies. Prey ingestion can be detected in otariids using stomach temperature transmitters [17], but short and variable retention times make long-duration deployments unreliable. Mandibular gape-angle sensors (IMASEN) can detect jaw opening in pinnipeds $[19,27,38]$, but feeding on small prey is often missed, and cabling may fail or affect the tagged animal over long durations.

With the miniaturization of biologging devices, heador jaw-mounted accelerometers have been investigated as a promising means to identify feeding or attempted prey capture in pinnipeds. These devices are compact, minimally invasive, relatively inexpensive, and have a midrange continuous sampling duration, making them an attractive alternative to other methods of feeding detection $[13,24,40]$. For appropriate use, however, acceleration signals must be validated against true feeding, as accelerations of the head and jaw are not limited to feeding motions $[11,29,35]$. Studies vary in their feeding identification criteria. The simplest techniques infer a feeding event when raw or filtered acceleration along one or two axes surpasses a threshold defined from a subset of training data (e.g., $[1,30])$. A variation of this method calculates the variance of those raw acceleration axes within a moving window and applies a similar threshold analysis to those data [13, 33, 35]. The simplicity of these methods makes them attractive but prone to false-positive feeding detections, as any sufficiently strong acceleration along the axis of analysis is identified as feeding [35]. A similar method using the triaxial norm Jerk (Norm of the differential of acceleration axes, $\mathrm{m} \mathrm{s}^{-3}$; [28] was found to reliably indicate prey capture and engulfment by a harbor seal in captive trials [40], and this method has been applied to harbor porpoises as well [39]. However, detection rates were not reported explicitly in those studies. Beyond using only a threshold, Skinner et al. [29] trained a model to detect feeding in Steller sea lions based on several acceleration measurements in 2-s windows, but relied only on acceleration data sampled along the surge axis $(32-64 \mathrm{~Hz})$. Like other studies, Skinner et al. [29] reported substantial false-positive detections ( 86 false-positive detections alongside 75 true-positive detections).

Here we test a method to detect prey capture with head-mounted accelerometers in California sea lions Zalophus californianus by searching for a diagnostic phrase of combined acceleration and Jerk data. There is not currently an acceleration-based method to remotely detect feeding in this species, thus the development of such a method should aid our ability to study fine-scale feeding patterns of this species in space and time. Our primary goal was to develop a selective automated feeding detection algorithm that minimized false-positive detections without substantially sacrificing true-positive detection accuracy. We also aimed to clarify the effect of accelerometer sampling rate on detection accuracy, as this could inform future efforts to balance detection accuracy against biologger battery life in wild deployments. Additionally, we sought to investigate relationships between prey length and characteristics of the detected prey capture signal phrase, since this too could help future work make inferences about relative prey size in wild deployments. Our final objective was to test the performance of the simpler triaxial Jerk method [40] in detecting the same feeding events, as detections from this method could more easily be relayed via telemetry in longer-term wild deployments.

\section{Methods}

\section{Experimental procedure}

Experiments were carried out at the SLEWTHS facility in Moss Landing, CA, with two trained adult sea lions (72 kg female 'Cali', $135 \mathrm{~kg}$ neutered male 'Nemo'). The subjects represented a wide range of movement variability within the species: Cali is small and could swim and maneuver rapidly in the pool, while Nemo moved more slowly due to his larger size and vision impairment (cataracts). Both were trained to wear a custom-built 1-mm neoprene head strap which held a small accelerometer (OpenTag, Loggerhead Instruments, Loggerhead.com) snugly against the dorsal surface of the skull (Fig. 1). In each experimental trial, the sea lion was sent by a trainer to swim across a seawater pool $(7.6 \times 4.6 \times 1.3 \mathrm{~m})$, capture and consume a dead fish of known total length (herring Clupea pallasii or capelin Mallotus villosus, $15.1-23.5 \mathrm{~cm}$ ), and return to the trainer. Fish were presented within $1 \mathrm{~m}$ of two underwater GoPro video cameras $\left(60\right.$ frames $\left.\mathrm{s}^{-1}\right)$ at the approximate depth of prey release and laterally offset in angle by 30 degrees to produce frames that overlapped spatially and recorded the entire trial. A third GoPro video camera recorded the entire experimental area from above water. Two types of trials were performed: prey capture trials with the accelerometer head-mounted as described above (Cali: $n=90$; Nemo: $n=67)$, and non-feeding 'control' trials to account for the acceleration signals of swimming and turning without prey capture (Cali: $n=75$; Nemo: $n=56$ ). During prey capture trials, sea lions displayed a tendency to anticipate the location of the dead fish. The control trials were introduced to account for the resulting consistent full-body movements; the sea lions were trained to swim the same route at the same pace, but no prey item was presented and they were called back to the trainer as they were approaching the target. 


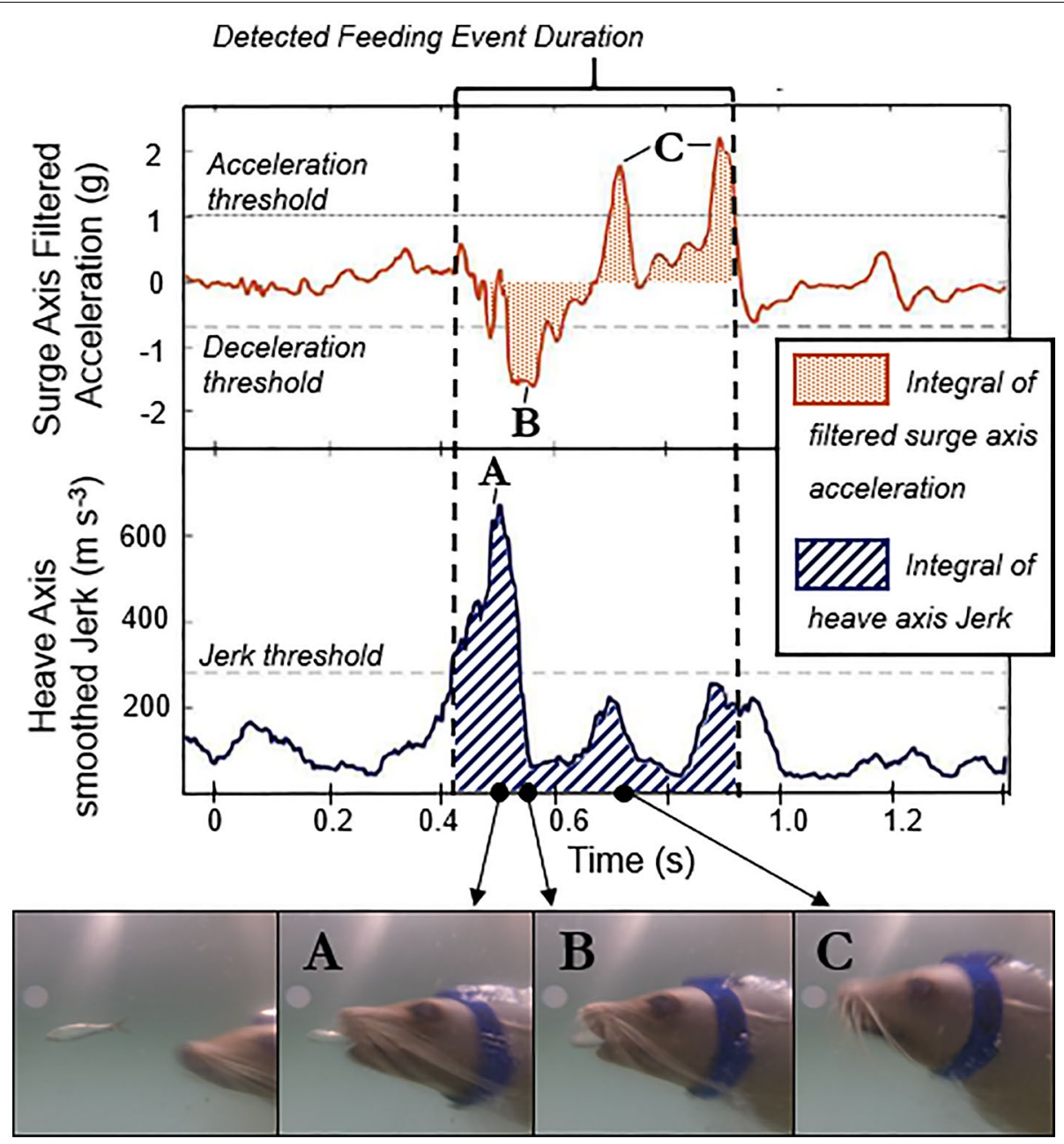

Fig. 1 Diagnostic signal phrase for detecting prey capture in California sea lions. Prey capture is detected from (A) a peak in heave axis smoothed Jerk data surpassing a threshold ('Jerk threshold) determined from sampling rate (shown in Fig. 2), resulting from a dorsal head tilt as the jaw opens; B a deceleration in estimated dynamic surge axis data surpassing $-0.7 \mathrm{~g}$ ('Deceleration threshold) within $0.2 \mathrm{~s}$ of the end of $A$, as the head retracts during suction or pierce feeding; and $\mathbf{C}$ a surge axis estimated dynamic acceleration surpassing $1.0 \mathrm{~g}$ ('Acceleration threshold) within $0.5 \mathrm{~s}$ of $B$, as the mouth closes and head rocks ventrally. The detected event duration and integrals of heave axis smoothed Jerk and surge axis estimated dynamic acceleration, which were evaluated for their relationships with prey length, are shown here graphically

\section{Data synchronizing and video analysis}

In all trials the OpenTag was set to record acceleration at $333 \mathrm{~Hz}$ with 16 bit resolution along 3 axes (heave, surge, sway). Static acceleration along each axis was calibrated before and after each experimental session (12 sessions, 6 to 23 trials per sea lion per session) by allowing the tag to sit steady in each of six stable resting orientations along each axis, recording maxima and minima for each axis, and scaling data to $[1,-1]$, the range expected due to gravity [37]. Care was taken to synchronize the OpenTag precisely with each GoPro: all GoPros continuously recorded the entire experimental session, capturing deliberate acceleration markers (each marker consisted of flicking the resting OpenTag four times in $10 \mathrm{~s}$ in view of all GoPros) before, between, and after trials in each session. Precise GoPro and OpenTag timestamps were recorded for each acceleration marker, and from these the relative drift between the OpenTag and GoPros was calculated and corrected between each marked point. All signal analyses were performed in MATLAB 2015b or 2016b (MathWorks, Natick, MA, USA).

Prior to analyzing acceleration data, framewise GoPro video analysis (Adobe Premier Pro) was used to record timestamps at (1) 5 frames after initial OpenTag submergence once the sea lion left the trainer; (2) initial mouth opening for prey capture (if applicable); (3) lower jaw closure following suction feeding, or the moment of raptorial biting (if applicable); (4) any repetitions of steps 2 
and 3 (in the case of prey handling following initial capture); (5) the approximate end of stereotyped prey capture head movements, marked by final closing of the jaw, and (6) 5 frames prior to the OpenTag visually surfacing from the water. The 5 -frame buffer in timestamps 1 and 6 was used to avoid acceleration artifacts caused by the tag nearing and breaking the water surface. In control trials, only timestamps 1 (submergence) and 6 (surfacing) were recorded.

Additionally, biomechanics of prey capture were noted from video analysis and used to inform the detector selection process (described below). Stereotyped feeding motions of the head, mouth, and neck were observed with reference to the movement imposed on the OpenTag. These biomechanical observations guided the order and timing of the acceleration patterns sought by the detector. For both sea lions, framewise video analysis revealed a consistent, stereotyped feeding motion consisting of (1) mouth opening; (2) a nearly concurrent rapid head retraction or stalling, peaking approximately during maximum gape; (3) a sharp forward head jolt as the jaw closed, and sometimes a rapid repetition of steps 1 through 3, if further prey handling was necessary to engulf prey.

\section{Training and testing the prey capture detector}

Video and acceleration data were divided into training and non-training datasets. The training dataset, which was composed of a random subset of each sea lion's prey capture trials (Cali: $n=24$, Nemo: $n=22$ ) and control trials (Cali: $n=16$; Nemo: $n=14$ ), was used to identify the combination of acceleration data patterns that most accurately identified feeding. We first visually inspected OpenTag data marked with video analysis timestamps, and identified a suite of patterns that appeared repeatedly, aligned with expectations from biomechanical video observations, and could potentially indicate prey capture. These patterns were then used in an iterative testing process to determine which pattern combinations most accurately identified true prey capture events in the training subset, as described in detail below. This process was applied to raw data $(333 \mathrm{~Hz})$, as well as raw data decimated to $200 \mathrm{~Hz}, 100 \mathrm{~Hz}, 50 \mathrm{~Hz}, 32 \mathrm{~Hz}, 20 \mathrm{~Hz}$, and $16 \mathrm{~Hz}$ to evaluate the consequences of lower sampling rate to prolong deployment time in the field.

For visual pattern inspection, accelerometer data were plotted for each trial in the training subset as both raw and estimated dynamic acceleration along each axis, the Jerk (product of sampling rate and the absolute value of the differential of acceleration) of each acceleration axis, and the triaxial norm Jerk (square-root of the sum of the squared differential of each axis, as defined in [40]). Estimated dynamic acceleration was calculated as the raw acceleration minus approximate static acceleration, with approximate static acceleration estimated by a moving mean using a window size of $0.5 \mathrm{~s}$. Triaxial norm Jerk and individual axis Jerk vectors were then smoothed over a window size of $0.05 \mathrm{~s}$ (sampling rate/20) to filter out highfrequency fluctuations due to either minor tag measurement error or potential accelerometer vibration resulting from the head strap. Visual inspection and comparison with concurrent video indicated that this window size produced an approximate static acceleration signal that estimated the changes in tag orientation during the rapid turning, rolling, and stalling motions that sometimes accompanied prey capture. All plots were overlain with timestamps recorded from video analysis for visual pattern inspection. This process identified a suite of possible indicative patterns (magnitude, duration, and directionality of signals) from each data form, yielding numerous combinations or 'phrases' of these patterns.

An iterative testing process (Additional file 1) was used to select the combination of acceleration and Jerk pattern that best identified true prey capture events (TP detection rate $=$ true detections/actual prey captures) in the prey capture training dataset, and ignored other motions associated with swimming or turning in the experimental training dataset (FP detection rate $=$ false detections/actual prey captures) and the control training dataset (Control FP detection rate $=$ control trial detections/control trials). This process occurred separately for Nemo and Cali. In each test iteration at each sampling rate, a different combination of thresholds and timing requirements were applied to the training subset as search criteria in custom-written MATLAB script, and the accuracy of prey capture detection (TP, FP, and Control FP detection rates, as defined above) was recorded. All pattern combinations identified from visual inspection were tested. For each sea lion at each sampling rate, the best pattern combination was manually selected using combined testing results from feeding trials (TP and FP detection rates) and control trials (Control FP detection rate) in the training dataset.

This iterative testing of the training datasets produced a consistent set of detection criteria that described the observed prey capture motion well across all sampling rates, for all feeding types observed (suction, pierce, and raptorial), in both Cali and Nemo (Fig. 1). The resulting detector required that data contain three components $(\mathrm{A}-\mathrm{C})$, each corresponding to a rapid motion during prey capture:

A. Spike in heave-axis Jerk An initial spike in heave-axis (dorso-ventral relative to the head) smoothed Jerk signal surpassing a threshold. This component traced a sharp increase in vertical acceleration due to mouth 
opening (step 1). Because Jerk values are dependent on sampling rate, it was necessary to describe this heave-axis Jerk threshold as a function of sampling rate. Threshold values were first determined separately for each sea lion, as part of the iterative testing process described above (Additional file 1). For both sea lions, a power curve best described the heaveaxis jerk threshold as a function of accelerometer analysis rate (Fig. 2).

B. Surge-axis deceleration Within $0.2 \mathrm{~s}$ of the end of (A), surge-axis (parallel to forward swimming direction) estimated dynamic deceleration must surpass $-0.7 \mathrm{~g}$ $\left(1 \mathrm{~g}=9.81 \mathrm{~m} \mathrm{~s}^{-2}\right)$. This component results from head retraction to facilitate full or partial prey engulfment (step 2).

C. Final surge-axis acceleration Following within $0.5 \mathrm{~s}$ of (B), surge-axis acceleration must surpass $1.0 \mathrm{~g}$. This component reflects a forward head movement concurrent with jaw closure (step 3).

The sequence of components $A-B$ must exceed $0.05 \mathrm{~s}$, to prevent detection of some rapid motions such as shaking or impact. In the case of prey handling, in which the sea lion does not successfully engulf prey during initial prey capture (steps $A-C$ ), the pattern of steps $A-C$ is repeated one or more times until prey is consumed. To be considered prey handling, any detected repetitions of $A-C$ must begin within $1 \mathrm{~s}$ of the end of the previous $A-C$ sequence; otherwise it is categorized as a new

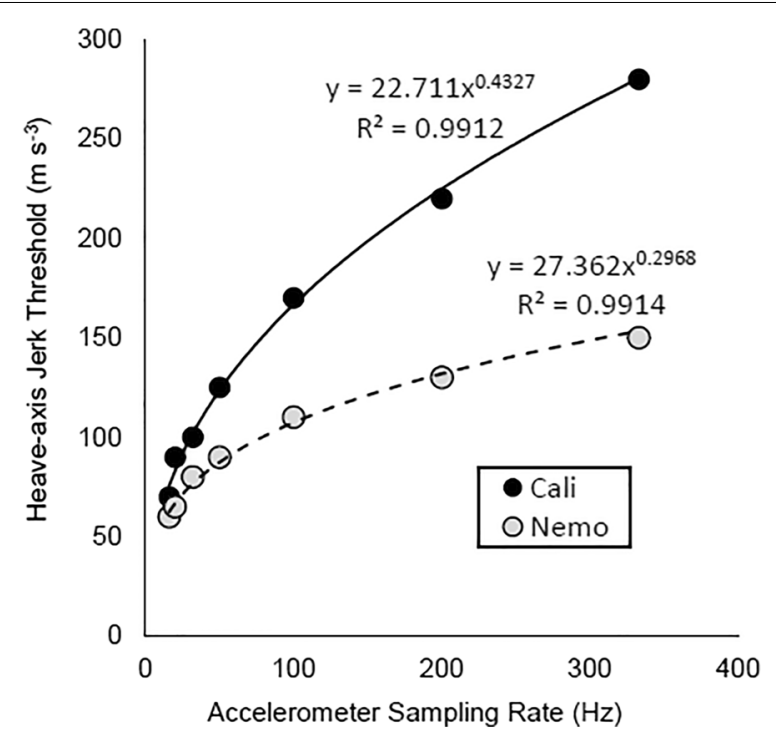

Fig. 2 Relationships between sampling rate and empirically determined heave axis smoothed Jerk thresholds. The curves for Cali and Nemo diverge as sampling rate increases, suggesting that model performance is more robust to individual differences at lower sampling rates feeding event. In this case of multiple $\mathrm{A}-\mathrm{C}$ sequences comprising one detection, the entire detected event must correspond to true feeding movements on video; detections that correspond to both feeding and clearly nonfeeding motions (e.g., prey searching) are categorized as both true positive and false positive.

Detectors were tested on the non-training trial and control subsets to determine their accuracy in identifying true-positive prey capture events (TP detection rate), ignoring false-positive detections during feeding trials (FP detection rate), and minimizing false-positive detections of the qualitatively similar rapid head and body movements in control trials (Control FP detection rate). Tests were conducted at 333, 200, 100, 50, 32, 20, and $16 \mathrm{~Hz}$.

To assess another promising prey capture detection method for comparison, we also calculated TP, FP, and Control FP detection rates for the same non-training datasets using the root mean square (RMS) of triaxial norm Jerk over an averaging window of $250 \mathrm{~ms}$ (square root of the mean of all squared triaxial norm Jerk values in the 250-ms window), a simpler method that works well in harbor seals and harbor porpoises [39, 40]. As with the detector, the optimal cutoff threshold was manually selected following iterative testing in training datasets (Additional file 1) for each sea lion and sampling rate.

Confident use with wild subjects requires a single general detector, but optimum heave-axis Jerk thresholds differed between sea lions (Fig. 2). Because Cali approached and captured prey more quickly and deliberately, she was judged to be the more representative model of a wild subject. We therefore tested the detectors calibrated for Cali against Nemo's data, across the full range of sampling rates, to determine the robustness of Cali's model to variation among subjects.

\section{Predicting prey size}

We investigated if variations in prey size were correlated with characteristics of the prey capture detection signal, and whether these characteristics varied between small and large prey size groupings. We reasoned that variations in prey size may produce differences in gape angle, feeding type (suction, pierce, or raptorial), speed of movement, and prey handling time prior to consumption $[9,10,15,20]$, and that these differences may affect prey capture detection signals [40]. Using the selected prey capture detectors for Nemo and Cali, a suite of possible indicators was calculated from acceleration and Jerk signals at each sampling rate; these indicators were (i) total prey capture duration; (ii) maximum heave-axis smoothed Jerk; (iii) maximum surge-axis estimated dynamic acceleration; (iv) the integral of heaveaxis smoothed Jerk, and (v) the integral of the absolute 
value of estimated dynamic surge-axis acceleration. We used simple (univariate) linear regressions to examine relationships between these indicators and prey length at each sampling rate, and we used T-tests (Student's or Welch's depending on equality of variance) to determine if each of these indicators could determine between smaller and larger prey size groups more generally. For both Cali and Nemo, prey shorter than mean prey length for that individual was categorized as 'small', and prey larger than mean prey length was categorized as 'large'

\section{Results}

\section{Prey capture detection accuracy}

Personalized detectors were accurate at mid-to-high sampling rates (Fig. 3A). For both Cali and Nemo, TP detection rate was high at $50-333 \mathrm{~Hz}(91.1-100 \%)$ and peaked at 100-200 $\mathrm{Hz}$ (Cali 100\%; Nemo 97.8\%). TP detection rate was also moderately high at $32 \mathrm{~Hz}(86.7-$ 91.5\%). A slight dip in TP detection rates at $333 \mathrm{~Hz}$ relative to 100 and $200 \mathrm{~Hz}$ is due to the need for a relatively stricter heave axis Jerk threshold to help filter out nonfeeding signals. TP detection rates were similar between Cali and Nemo across all sampling rates, though slightly higher in Cali (except $333 \mathrm{~Hz}$ ). There were no feeding trial FP detections ( $0 \%$ FP detection rate) for either sea lion, at any sampling rate, indicating that propulsive strokes and head movements while searching for prey were not mistaken for feeding.

Cali's detector identified Nemo's feeding quite accurately between 32 and $100 \mathrm{~Hz}$ (Fig. 3A). TP detection rate peaked at $50 \mathrm{~Hz}$ (91.11\%), equivalent to Nemo's detector at the same sampling rate. At $50 \mathrm{~Hz}$ and below, TP detection rates tracked those of Nemo's detector. From 100 to $333 \mathrm{~Hz}$, TP detection rates decreased with higher sampling rate. This is expected given individual differences: at higher sampling rates, it becomes increasingly difficult for Nemo's heave axis Jerk data to reach the threshold set by Cali's detection model (Fig. 2).

Control FP detection rates were below 5\% across all sampling rates for both animals, including when Cali's detector was applied to Nemo's data (Fig. 3A; Cali: $0-1.51 \%$, Nemo: $0-4.76 \%$ ). The control trials that were falsely detected were qualitatively similar to prey capture in head movement: in these trials, and in several others that were not detected, the sea lion stationed at a target (rapid deceleration) and then actively pushed the target (acceleration) before returning to the trainer.

In contrast to the detectors, the triaxial RMS Jerk method [40] produced high TP detection rates (91.4-100\%), but elevated Control FP detection rates (15-66.7\%) and FP detection rates (up to 17.2\%), at all sampling rates (Fig. 3B). TP detection rate did not decrease at lower sampling rates, but Control FP
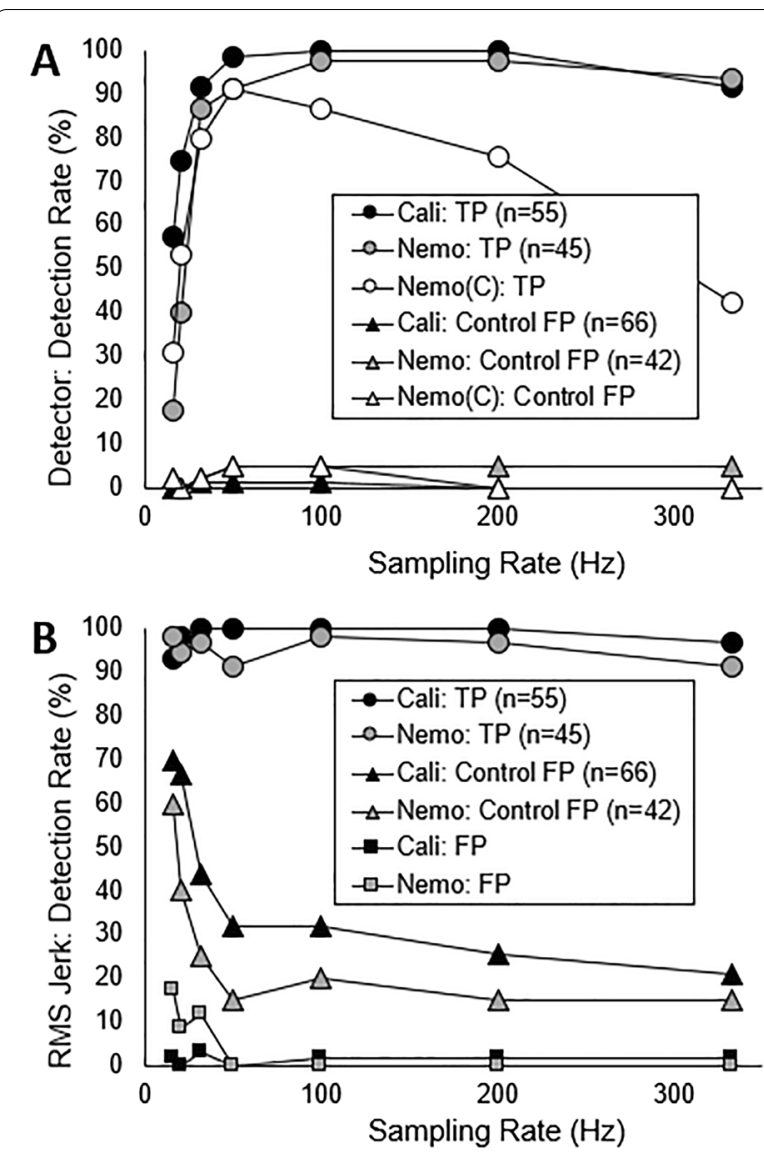

Fig. 3 True positive (TP), control trial false-positive (Control FP), and feeding trial false-positive (FP) detection rates in the test datasets (excluding the training datasets) across sampling rate. Control FP detection rates were found by dividing the number of false-positive detections during control trials by the number of control trials. TP and FP detection rates were found by dividing the numbers of true and false-positive detections during feeding trials by the number of feeding trials. TP, Control FP, and FP detection rates are expressed here as percentages (i.e., multiplied by 100). TP and Control FP detection rates of Nemo's data using Cali's detector are labeled 'Nemo (C)'. Shown are detection rates using (A) the detectors (Fig. 1) optimized for each sea lion, and B) RMS Jerk summed over a 250-ms window with individually optimized thresholds (adjusted from Ydesen et al. [40] to produce detection rates)

detection rate did increase substantially below $50 \mathrm{~Hz}$. Unlike the detector method, non-feeding movements were falsely detected during feeding trials (Fig. 3B) with the RMS Jerk method.

\section{Predicting prey length}

Prey length was related to some indicators quantified in TP detections by the detector, but results varied between Cali and Nemo and across sampling rates (Table 1, Fig. 4). In Cali's data, prey capture duration, as well as integrated heave-axis Jerk and integrated absolute value 
Table 1 Relationships between prey length or prey length grouping and characteristics of true-positive detections

\begin{tabular}{|c|c|c|c|c|c|c|c|c|c|c|c|c|}
\hline \multirow[t]{2}{*}{ Sampling rate } & \multirow[t]{2}{*}{ Test } & \multirow[t]{2}{*}{ DF } & \multicolumn{2}{|c|}{$\begin{array}{l}\text { Prey capture } \\
\text { duration }\end{array}$} & \multicolumn{2}{|c|}{$\begin{array}{l}\text { Max. heave } \\
\text { Jerk }\end{array}$} & \multicolumn{2}{|c|}{$\begin{array}{l}\text { Max. surge } \\
\text { deceleration }\end{array}$} & \multicolumn{2}{|c|}{ Heave Jerk integral } & \multicolumn{2}{|l|}{$\begin{array}{l}\text { Surge accel. } \\
\text { integral }\end{array}$} \\
\hline & & & $p$ & $R^{2}$ & $p$ & $R^{2}$ & $p$ & $R^{2}$ & $p$ & $R^{2}$ & $p$ & $R^{2}$ \\
\hline \multicolumn{13}{|l|}{ CALI } \\
\hline \multirow[t]{2}{*}{$333 \mathrm{~Hz}$} & Lin. Reg & 54 & $<0.0001^{*}$ & 0.254 & 0.214 & 0.029 & 0.098 & 0.051 & $0.0003^{*}$ & 0.221 & $<0.0001^{*}$ & 0.346 \\
\hline & T-test & 53 & $<0.0001^{*}$ & & 0.419 & & 0.334 & & $0.008^{*}$ & & $<0.0001 *$ & \\
\hline \multirow[t]{2}{*}{$200 \mathrm{~Hz}$} & Lin. Reg & 54 & $<0.0003^{*}$ & 0.226 & 0.351 & 0.016 & 0.079 & 0.057 & $<0.0001 *$ & 0.271 & $<0.0001 *$ & 0.399 \\
\hline & T-test & 53 & $<0.0001^{*}$ & & 0.648 & & 0.294 & & $0.002^{*}$ & & $<0.0001 *$ & \\
\hline \multirow[t]{2}{*}{$100 \mathrm{~Hz}$} & Lin. Reg & 55 & $<0.0001^{*}$ & 0.284 & 0.487 & 0.009 & 0.056 & 0.066 & $<0.0001 *$ & 0.269 & $<0.0001 *$ & 0.435 \\
\hline & T-test & 54 & $<0.0001^{*}$ & & 0.772 & & 0.253 & & $0.003^{*}$ & & $<0.0001 *$ & \\
\hline \multirow[t]{2}{*}{$50 \mathrm{~Hz}$} & Lin. Reg & 54 & $<0.0001^{*}$ & 0.357 & 0.224 & 0.028 & 0.221 & 0.028 & $<0.0001^{*}$ & 0.28 & $<0.0001 *$ & 0.482 \\
\hline & T-test & 53 & $<0.0001^{*}$ & & 0.660 & & 0.348 & & $0.004^{*}$ & & $<0.0001 *$ & \\
\hline \multirow[t]{2}{*}{$32 \mathrm{~Hz}$} & Lin. Reg & 50 & $<0.0001^{*}$ & 0.314 & 0.292 & 0.025 & 0.275 & 0.024 & $0.002 *$ & 0.18 & $0.0004^{*}$ & 0.225 \\
\hline & T-test & 49 & $<0.0001^{*}$ & & 0.938 & & 0.695 & & $0.019^{*}$ & & $0.012^{*}$ & \\
\hline \multirow[t]{2}{*}{$20 \mathrm{~Hz}$} & Lin. Reg & 34 & 0.243 & 0.041 & 0.372 & 0.024 & 0.89 & $<0.001$ & $0.026^{*}$ & 0.142 & $0.027^{*}$ & 0.14 \\
\hline & T-test & 33 & 0.108 & & 0.609 & & 0.756 & & $0.016^{*}$ & & $0.002 *$ & \\
\hline \multirow[t]{2}{*}{$16 \mathrm{~Hz}$} & Lin. Reg & 25 & 0.157 & 0.082 & 0.416 & 0.028 & 0.667 & 0.008 & $0.005^{*}$ & 0.285 & $0.006^{*}$ & 0.277 \\
\hline & T-test & 24 & 0.316 & & 0.155 & & 0.994 & & $0.004^{*}$ & & $0.013^{*}$ & \\
\hline \multicolumn{13}{|l|}{ NEMO } \\
\hline \multirow[t]{2}{*}{$333 \mathrm{~Hz}$} & Lin. Reg & 41 & 0.098 & 0.067 & 0.841 & 0.001 & 0.151 & 0.051 & 0.084 & 0.073 & 0.111 & 0.062 \\
\hline & T-test & 40 & $0.018^{*}$ & & 0.837 & & 0.066 & & 0.079 & & 0.097 & \\
\hline \multirow[t]{2}{*}{$200 \mathrm{~Hz}$} & Lin. Reg & 43 & 0.108 & 0.06 & 0.774 & 0.002 & 0.113 & 0.059 & $0.044^{*}$ & 0.093 & 0.078 & 0.072 \\
\hline & T-test & 42 & $0.036^{*}$ & & 0.924 & & 0.062 & & 0.095 & & 0.123 & \\
\hline \multirow[t]{2}{*}{$100 \mathrm{~Hz}$} & Lin. Reg & 43 & 0.087 & 0.068 & 0.838 & 0.001 & 0.114 & 0.059 & $0.049^{*}$ & 0.089 & $0.049^{*}$ & 0.089 \\
\hline & T-test & 42 & $0.028^{*}$ & & 0.949 & & 0.079 & & 0.125 & & 0.086 & \\
\hline \multirow[t]{2}{*}{$50 \mathrm{~Hz}$} & Lin. Reg & 38 & 0.234 & 0.038 & 0.387 & 0.02 & 0.2 & 0.044 & 0.165 & 0.051 & 0.244 & 0.037 \\
\hline & T-test(W) & $37(26)$ & 0.090 & & 0.294 & & $0.039^{*}$ & & 0.141 & & 0.144 & \\
\hline \multirow[t]{2}{*}{$32 \mathrm{~Hz}$} & Lin. Reg & 18 & 0.105 & 0.147 & 0.36 & 0.05 & 0.64 & 0.013 & 0.169 & 0.108 & 0.238 & 0.081 \\
\hline & T-test & 17 & $0.014^{*}$ & & 0.905 & & 0.312 & & 0.208 & & 0.166 & \\
\hline \multirow[t]{2}{*}{$20 \mathrm{~Hz}$} & Lin. Reg & 17 & 0.619 & 0.016 & 0.528 & 0.025 & 0.06 & 0.205 & 0.697 & 0.009 & 0.791 & 0.005 \\
\hline & T-test (W) & $16(10)$ & $0.026^{*}$ & & 0.151 & & 0.192 & & 0.069 & & 0.196 & \\
\hline \multirow[t]{2}{*}{$16 \mathrm{~Hz}$} & Lin. Reg & 7 & 0.386 & 0.127 & 0.127 & 0.343 & 0.782 & 0.014 & 0.364 & 0.139 & 0.936 & 0.001 \\
\hline & T-test & 6 & 0.335 & & 0.186 & & 0.732 & & 0.734 & & 0.881 & \\
\hline
\end{tabular}

Lin. Reg. indicates simple linear regressions. T-test refers to 2-sample Student's t-tests assuming equal variance, comparing among large and small prey length groupings. T-test $(W)$ indicates that Welch's t-tests were used when variance was unequal among large and small prey length grouping. Df numbers in parentheses are degrees of freedom in Welch's t-tests. Df varies with sampling rate within individual because only data from true-positive detections were included in statistical analyses. T-test $\mathrm{df}=(n-2)$ and Lin. Reg. $\mathrm{df}=(n-1)$

Bold, italicized font and the * symbol denotes either a significant relationship (Lin. Reg.) or a significant difference among prey length group (T-test or T-test(W))

of surge-axis estimated dynamic acceleration signals increased significantly with prey length, but these and other linear relationships were mostly absent in Nemo's data (simple linear regressions; Fig. 4, Table 1).

When data were grouped by prey length, larger fish were found to produce significantly longer detected prey capture durations across a majority of sampling rates in both Cali and Nemo (Student's or Welch's T-tests; Fig. 4, Table 1). The larger prey group also produced significantly larger integrated heave-axis Jerk and integrated surge-axis estimated dynamic acceleration signals in
Cali's data across sampling rate, but not in Nemo's data (Fig. 4, Table 1).

\section{Discussion}

Using supercranial acceleration data at mid-to-high sampling rates (32 Hz or higher), the stereotyped head movements of prey capture can be identified with high accuracy in California sea lions. While similar acceleration-based procedures for detecting prey capture (or attempted prey capture) in pinnipeds exist, the detector outlined here builds upon these methods by searching 


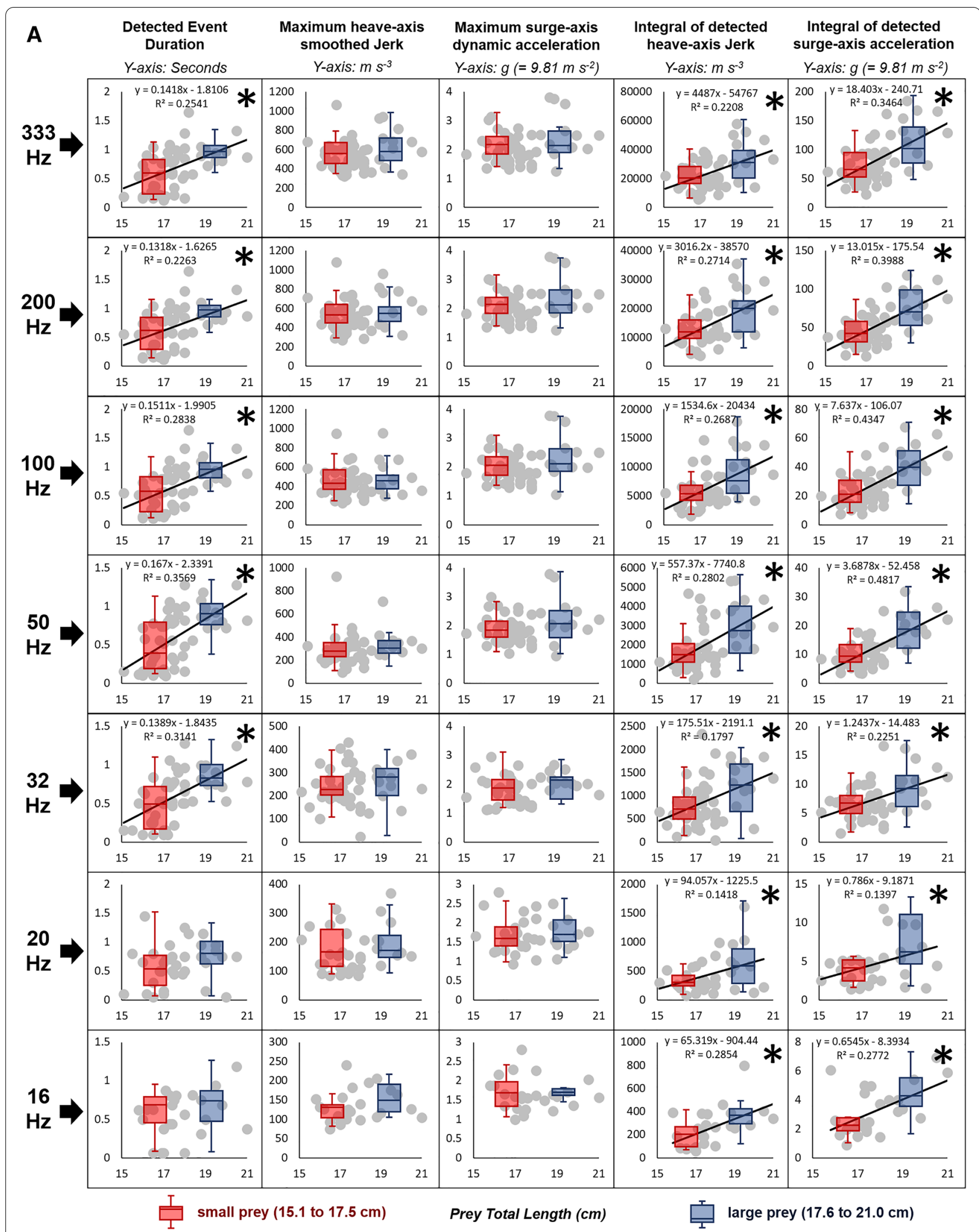

Fig. 4 Relationships between prey length and signal characteristics for Cali (A) and Nemo (B). Y-axis units are indicated at the top of each column. Raw signal characteristic data from TP detections at each sampling rate are shown with grey dots. Significant univariate linear relationships are shown with black lines through the raw data. Box plots comparing signal characteristics among small (red) and large (blue) prey length groupings are overlain on each plot; asterisks indicate plots where these groupings differed significantly 


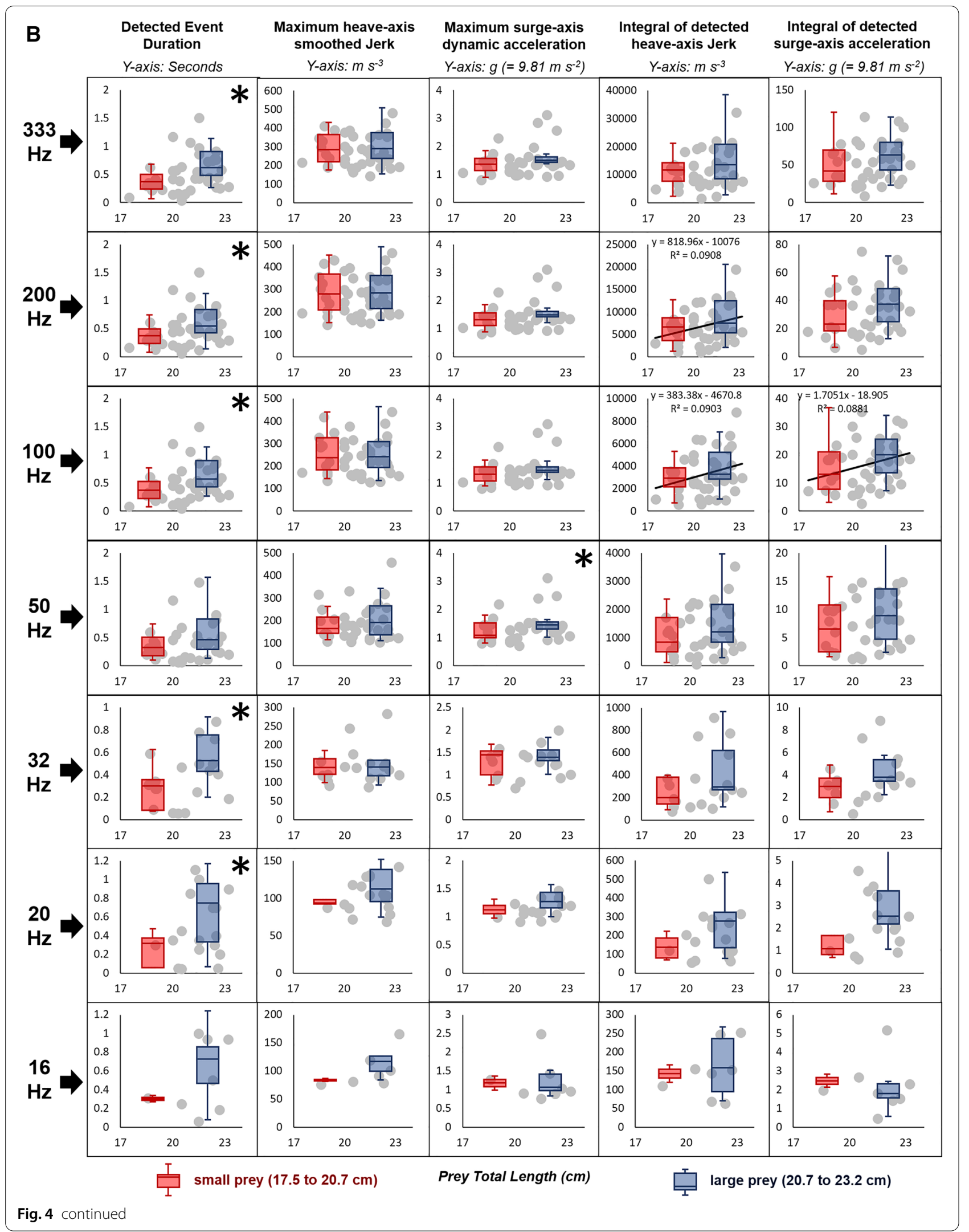


for a specific phrase of signals to minimize FP detections while maintaining high TP detection rates.

For California sea lions, a selective signal detector appears necessary to discern feeding from other movements. Though triaxial Jerk (a single signal surpassing a threshold) was sufficient for high TP detection (Fig. 3B), searching for a specific signal combination for use in a detector (Fig. 1) was key to minimizing FP detection. Precisely synchronized acceleration data and high-speed video allowed us to isolate acceleration and Jerk data patterns, occurring at time scales of tens to hundreds of milliseconds, that reliably aligned with head movements during prey capture and thus allowed detectors of this detail.

Our finding that specific, biologically informed pattern recognition decreases false positives is consistent with the observed sources of detection error in previous studies in otariids. Skinner et al. [29] found that dynamic surge-axis acceleration (at 32 or $64 \mathrm{~Hz}$ ) correctly detected $>80 \%$ of actual fish capture attempts (75 of 92), but also made 86 FP detections over the same trial period. Eighty-five of their 86 FP detections occurred while chasing fish, highlighting the need for specific pattern recognition to better discern between high-acceleration behaviors. Similarly, Viviant et al. [33] observed that many of the false-positive detections in their study, which used the calculated variance of individual-axis filtered acceleration from both head- and jaw-mounted accelerometers on captive Steller sea lions, reflected occasions of mouth opening unrelated to feeding.

Sampling rate influenced the detector's accuracy. While FP and Control FP detection rates remained low across all sampling rates, TP detection rate decreased sharply below $32 \mathrm{~Hz}$, due to loss of details in the acceleration signal and corresponding dampening of the heave-axis Jerk signal (Fig. 5). Because key signals required by the detector (i.e., Fig. 1) occurred over approximately 0.05 to $0.2 \mathrm{~s}$ each, sampling at a low rate resulted in acceleration and particularly Jerk signals that mischaracterized the true head movements. At our highest sampling rates $(333 \mathrm{~Hz})$, however, the detector was less robust to inter-individual differences (Fig. 3A). These combined results suggest a 'sweet spot' at around $50 \mathrm{~Hz}$ for this detector.

The duration of the detected signal appeared to be able to differentiate, on average, between relatively smaller and larger prey at moderate sampling rates in both sea lions (Fig. 4, Table 1). This is consistent with results from a captive method validation with elephant seals (Mirounga angustirostris) that found larger prey size groups elicited a greater number of prey capture signals [1]. Their results, alongside those presented here, likely follow from the extended prey handling

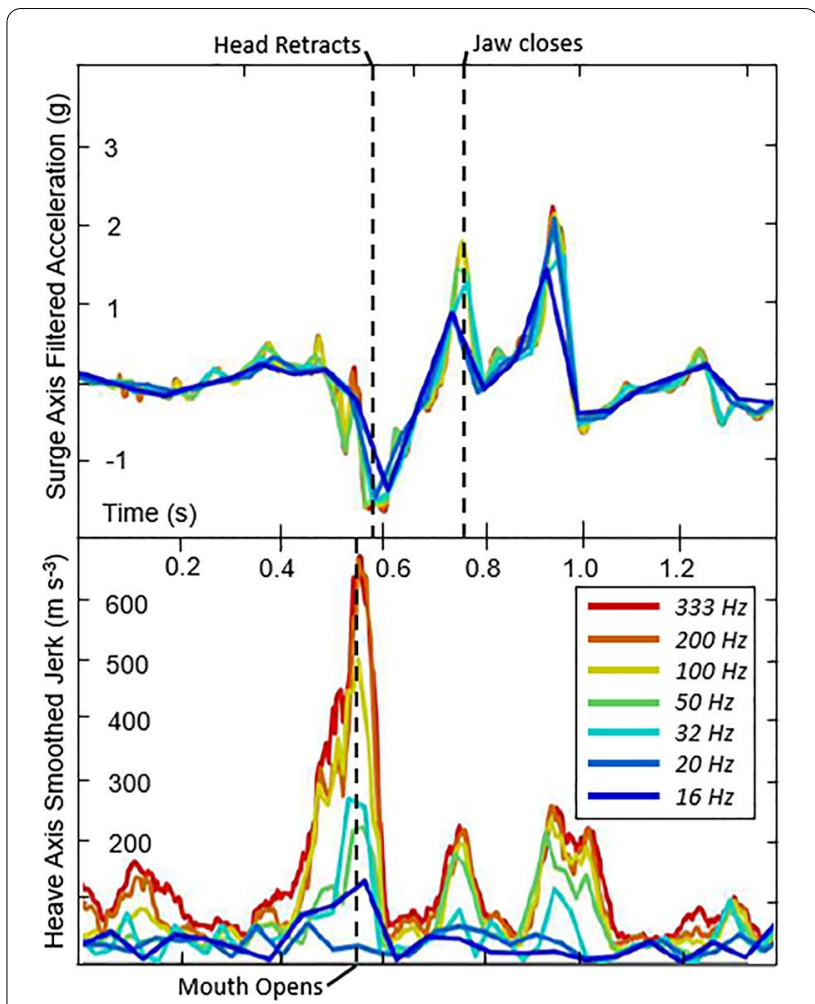

Fig. 5 The effect of sampling rate on signals used in the detector. Timing of key prey capture movements as noted from video analysis are shown with dotted lines. Surge axis estimated dynamic acceleration signals are relatively conserved, whereas heave axis smoothed Jerk signals are strongly attenuated by decreased sampling rate, with timing and magnitude particularly obscured below $32 \mathrm{~Hz}$

needed to capture and consume larger prey, as suggested by Ydesen et al. [40]. Other signal characteristics tested in this study were either not different among prey length categories, or showed inconclusive trends due to inter-individual differences (Fig. 4, Table 1). In particular, the greater noise in Nemo's data overshadowed the effect of prey length on the integral of detected heaveaxis Jerk and the integral of detected surge-axis acceleration, which were among the strongest relationships in Cali's data (Fig. 4, Table 1. The strength of those trends in Cali's data may hint that larger prey also elicit larger magnitude detection signals; however, the integrals of heave-axis Jerk and surge-axis acceleration are influenced by the detected prey capture duration (Fig. 1), and maximum detected surge-axis acceleration and heave-axis Jerk values did not vary with prey length nor among size classes (Fig. 4). It is likely, too, that the restricted prey lengths available for this study limited our ability to detect effects of prey length in some signal characteristics. 


\section{Limitations and use on wild otariids}

This detector recognizes the stereotyped signal patterns of initial prey capture and should therefore be applicable to studies of feeding patterns in wild California sea lions. As with any accelerometry-based feeding detection method, video validation in wild subjects will be needed to confirm the method's performance in wild settings. Captive studies like this are logistically limited in sample size (e.g., 2 sea lions here) and therefore in scope of inference. In addition, other limitations exist when applying methods validated in controlled settings to wild animals; these generally reflect behavioral differences between subjects, differences in prey, and settings within the detector.

Individual subjects may differ in their ideal detector parameters [35]. In our case, prey capture detections were optimized in a controlled setting with personalized heave-axis Jerk thresholds (Fig. 2), reflecting differences among individuals. Despite this, Cali's personalized detector accurately detected Nemo's actual feeding when used at moderate sampling rates $(32-100 \mathrm{~Hz})$, supporting the potential use of a single general detector at moderate sampling rates $(\sim 50 \mathrm{~Hz})$ to detect feeding or attempted feeding by wild California sea lions. Cali's detector is freely available (https://github.com/masonrcole/CSL_ Feeding_Detector). In future applications on wild subjects, detector parameters would ideally be validated against video and potentially adjusted on either a universal or individual basis (as in [35]) to maximize detection accuracy and assess the use of universal detection parameters.

Validation using dead prey in a controlled environment allows for detailed isolation of prey capture signals, but yields a limited range of observations. Vigorous prey pursuit or extended prey handling could produce acceleration signals not observed during captive validations with dead prey [11, 29, 35]. While we could not test these scenarios, the strict criteria used in our detector should help to minimize FP detection rates by selecting for biologically informed patterns in addition to minimum signal magnitudes.

Larger and live prey in wild settings should not negatively affect this detector's TP detection rate, though it is unknown how these factors will affect the FP detection rate, as mentioned above. Our detector will infer prey capture so long as the minimum requirements are met (e.g., Fig. 1), meaning accelerations that occur in addition to the stereotyped initial prey capture signal will not prevent true-positive feeding detection, but could produce a false positive in addition to the true-positive detection depending on context. Within pinniped species, the head and jaw kinematics of initial prey capture (suction, pierce, or raptorial feeding) comprise a narrow range of stereotyped movements [9, 10, 15, 20]. Our prey size prediction trends indicate that larger (though still small) prey elicit temporally extended, but not fundamentally different, acceleration and Jerk signals. Our use of small dead prey ensures that even feeding acceleration signals on small prey are detected. We expect that larger and actively swimming prey will produce acceleration signals that meet the detector's requirements, given that these requirements stem from consistent motions (mouth opening, head deceleration, and mouth closing) that we observed to occur in each feeding type (suction, pierce, and raptorial), even if these acceleration signals occur among other accelerations associated with chasing and prey handling. Acceleration and Jerk signals from larger and live prey will likely exceed the magnitude of signals from small dead prey, as previously observed (e.g., [40]).

Because our prey sizes were restricted, we could not fully validate relationships between detection signals and prey size. Larger prey will likely require more handling time, including tearing at the surface $[9,10]$. While we expect our detector to identify initial prey capture, as outlined above, we could not calibrate or test the ability of our detector to identify these instances of prey handling that are temporally distinct from prey capture. Video validation with wild subjects (e.g., [35]) would be necessary to assess the influence of these behaviors on FP detection rate. Additionally, like other methods, our detector is likely to detect a subset of attempted but unsuccessful prey captures [29, 35]. Finally, this detector should be applied in appropriate diving context: breaking the air-water barrier and shallow-water conspecific interactions could produce acceleration signals that mimic prey capture by chance. These shallow or surface behaviors could be filtered out in wild studies by applying a depth minimum (e.g., $[1,25,35])$.

\section{Conclusions}

Knowledge of feeding patterns is key to understanding an animal's ecological role and energetic trade-offs, yet methods to identify prey capture by marine mammals, and otariids in particular, remain relatively inaccurate or expensive. Fine-scale feeding data inform our understanding of ecosystem impact, ecological niche, and rates of energetic gain from prey, the latter of which further affects reproductive success and ultimately population trends $[6,12-14,21-23,31,32]$.

The analysis introduced and validated in this study accurately detected feeding by California sea lions in a controlled setting, using strict detection requirements to help minimize false-positive detections. The detector developed here was most accurate at moderate-to-high sampling rates $(32-333 \mathrm{~Hz})$, and was robust to interindividual variability at moderate sampling rates, with 
best performance at $50 \mathrm{~Hz}$. Furthermore, we found that larger prey elicited longer detected prey capture duration than smaller prey at most moderate-to-high sampling rates (both sea lions $32-333 \mathrm{~Hz}$, except Nemo $50 \mathrm{~Hz}$ ). This detector therefore offers a low-cost and mid-duration potential means to observe the temporal and spatial patterns of wild California sea lion feeding events, along with a captive-validated metric (prey capture duration) to observe how relative prey length may vary in individual subjects across habitat, depth, foraging strategy, or the duration of a foraging trip or dive bout. We also assessed the accuracy of the triaxial Jerk analysis [40] on California sea lions in the same feeding trials, and found high TP rates at all sampling rates with low-to-moderate FP rates in control trials above $50 \mathrm{~Hz}$. Although these control trial FP rates were elevated relative to the detector developed in this study, the simpler detection criteria could make the triaxial Jerk method a favorable option for long-duration deployments that rely on on-board signal processing and low-bandwidth telemetry of processed data. Overall, we propose that these methods can potentially improve our ability to pinpoint feeding by wild California sea lions.

\section{Abbreviations}

FP: False positive; g: Acceleration due to gravity; $9.81 \mathrm{~m} \mathrm{~s}^{-1}$; RMS: Root mean squared; TP: True positive.

\section{Supplementary Information}

The online version contains supplementary material available at https://doi, org/10.1186/s40317-021-00267-7.

Additional file 1: Iterative testing ranges and intervals for signal thresholds and timing patterns that were identified from video observation.

\section{Acknowledgements}

We thank the trainers and staff at SLEWTHS for exceptional logistical help, specific training, and accommodation. We thank Peter Madsen for lending OpenTags and for helpful comments on the manuscript. We also thank the MLML Vertebrate Ecology Lab for support and feedback throughout the project.

\section{Authors' contributions}

MRC and BIM conceived and designed the study. MRC, SS and JAZ conducted fieldwork. SS and JAZ trained the sea lions. MRC processed data, performed data analysis, and wrote the manuscript. MRC, BIM and JAZ reviewed and edited the manuscript. All authors read and approved the final manuscript.

\section{Funding}

This research was supported by the COAST Graduate Student Research Award (CSUCOAST-COLMAS-SJSU-AY1617 awarded to M.R.C.), the H.T. Harvey Memorial Research Fellowship, the MLML Scholar Award, the S.J.S.U. Archimedes Scholarship, and the Dr. Earl H. Myers \& Ethel M. Myers Oceanographic and Marine Biology Trust (all awarded to M.R.C.). Additional support was provided by the Office of Naval Research (Award number \#: N000141612852 awarded to B.I.M.)

\section{Availability of data and materials}

The datasets used and/or analyzed during the current study are available from the corresponding author on reasonable request.

\section{Declarations}

Ethics approval and consent to participate

Research was conducted under San Jose State University IACUC protocol numbers 1009 and 1027, and under APHIS permit numbers 93-c-0626 and 93-R-0427.

\section{Consent for publication \\ Not applicable.}

\section{Competing interests}

No competing interests declared.

Received: 30 December 2020 Accepted: 12 October 2021

Published online: 21 October 2021

\section{References}

1. Adachi T, Hückstädt LA, Tift MS, Costa DP, Naito Y, Takahashi A. Inferring prey size variation from mandible acceleration in northern elephant seals. Mar Mamm Sci. 2019;35(3):893-908.

2. Bowen WD, Tully D, Boness DJ, Bulheier BM, Marshall GJ. Prey-dependent foraging tactics and prey profitability in a marine mammal. Mar Ecol Prog Ser. 2002;244:235-45

3. Costa DP, Gales NJ. Energetics of a benthic diver: seasonal foraging ecology of the Australian sea lion, Neophoca cinerea. Ecol Monog. 2003;73(1):27-43.

4. Davis RW, Fuiman LA, Williams TM, Collier SO, Hagey WP, Kanatous SB, Kohin S, Horning M. Hunting behavior of a marine mammal beneath the Antarctic fast ice. Science. 1999;283(5404):993-6.

5. Estes JA, Jameson RJ, Rhode EB. Activity and prey election in the sea otter: influence of population status on community structure. Am Nat. 1982;120(2):242-58.

6. Estes JA, Steneck RS, Lindberg DR. Exploring the consequences of species interactions through the assembly and disassembly of food webs: a Pacific-Atlantic comparison. Bull Mar Sci. 2013;89(1):11-29.

7. Feldkamp SD, DeLong RL, Antonelis GA. Diving patterns of California sea lions, Zalophus californianus. Can J Zool. 1989;67(4):872-83.

8. Goldbogen JA, Calambokidis J, Oleson E, Potvin J, Pyenson ND, Schorr G, Shadwick RE. Mechanics, hydrodynamics and energetics of blue whale lunge feeding: efficiency dependence on krill density. J Exp Biol. 2011;214(1):131-46.

9. Hocking DP, Fitzgerald EM, Salverson M, Evans AR. Prey capture and processing behaviors vary with prey size and shape in Australian and subantarctic fur seals. Mar Mamm Sci. 2016;32(2):568-87.

10. Hocking DP, Ladds MA, Slip DJ, Fitzgerald EM, Evans AR. Chew, shake, and tear: prey processing in Australian sea lions (Neophoca cinerea). Mar Mamm Sci. 2017;33(2):541-57.

11. Iwata T, Sakamoto KQ, Takahashi A, Edwards EW, Staniland IJ, Trathan PN, Naito Y. Using a mandible accelerometer to study fine-scale foraging behavior of free-ranging Antarctic fur seals. Mar Mamm Sci. 2012;28(2):345-57.

12. Jeglinski JW, Goetz KT, Werner C, Costa DP, Trillmich F. Same sizesame niche? Foraging niche separation between sympatric juvenile Galapagos sea lions and adult Galapagos fur seals. J Anim Ecol. 2013;82(3):694-706.

13. Jeanniard-du-Dot T, Trites AW, Arnould JP, Guinet C. Reproductive success is energetically linked to foraging efficiency in Antarctic fur seals. PLoS ONE. 2017;12(4):e0174001.

14. Kelaher BP, Tan M, Figueira WF, Gillanders BM, Connell SD, Goldsworthy SD, Hardy N, Coleman MA. Fur seal activity moderates the effects of an Australian marine sanctuary on temperate reef fish. Biol Conserv. 2015;182:205-14. 
15. Kienle SS, Hermann-Sorensen H, Costa DP, Reichmuth C, Mehta RS. Comparative feeding strategies and kinematics in phocid seals: suction without specialized skull morphology. J Exp Biol. 2018;221(15):jeb179424.

16. Kooyman GL. Genesis and evolution of bio-logging devices: 1963-2002. Mem Natl Inst Polar Res. 2004;58:15-22.

17. Kuhn CE, Costa DP. Identifying and quantifying prey consumption using stomach temperature change in pinnipeds. J Exp Biol. 2006;209(22):4524-32.

18. Le Boeuf BJ, Naito Y, Asaga T, Crocker D, Costa DP. Swim speed in a female northern elephant seal: metabolic and foraging implications. Can J Zool. 1992;70(4):786-95.

19. Liebsch N, Wilson RP, Bornemann H, Adelung D, Plötz J. Mouthing off about fish capture: jaw movement in pinnipeds reveals the real secrets of ingestion. Deep Sea Res Part II Top Stud Oceanogr. 2007;54(3-4):256-69.

20. Marshall CD, Rosen D, Trites AW. Feeding kinematics and performance of basal otariid pinnipeds, Steller sea lions (Eumetopias jubatus), and northern fur seals (Callorhinus ursinus): implications for the evolution of mammalian feeding. J Exp Biol. 2015;218(20):3229-40.

21. Melin SR, DeLong RL, Siniff DB. The effects of El Niño on the foraging behavior of lactating California sea lions (Zalophus californianus californianus) during the nonbreeding season. Can J Zool. 2008;86(3):192-206.

22. McClatchie S, Field J, Thompson AR, Gerrodette T, Lowry M, Fiedler PC, Watson W, Nieto KM, Vetter RD. Food limitation of sea lion pups and the decline of forage off central and southern California. R Soc Open Sci. 2016;3(3):150628

23. McHuron EA, Robinson PW, Simmons SE, Kuhn CE, Fowler M, Costa DP. Foraging strategies of a generalist marine predator inhabiting a dynamic environment. Oecologia. 2016;182(4):995-1005.

24. Naito Y. How can we observe the underwater feeding behavior of endotherms? Polar Sci. 2007;1(2-4):101-11.

25. Naito Y, Costa DP, Adachi T, Robinson PW, Fowler M, Takahashi A. Unravelling the mysteries of a mesopelagic diet: a large apex predator specializes on small prey. Funct Ecol. 2013;27(3):710-7.

26. Parrish FA, Marshall GJ, Littnan C, Heithaus M, Canja S, Becker B, Braun R, Antoneijs GA. Foraging of juvenile monk seals at French Frigate Shoals, Hawaii. Mar Mamm Sci. 2005;21(1):93-107.

27. Ropert-Coudert Y, Kato A, Liebsch N, Wilson RP, Muller G, Baubet E. Monitoring jaw movements: a cue to feeding activity. Game Wildl Sci. 2004;20(4):1-19.

28. Simon M, Johnson M, Madsen PT. Keeping momentum with a mouthfu of water: behavior and kinematics of humpback whale lunge feeding. J Exp Biol. 2012;215(21):3786-98.

29. Skinner JP, Norberg SE, Andrews RD. Head striking during fish capture attempts by Steller sea lions and the potential for using head surge acceleration to predict feeding behavior. Endanger Species Res. 2009;10:61-9.

30. Suzuki I, Naito Y, Folkow LP, Miyazaki N, Blix AS. Validation of a device for accurate timing of feeding events in marine animals. Polar Biol. 2009;32(4):667-71.
31. Villegas-Amtmann S, Costa DP, Tremblay Y, Salazar S, Aurioles-Gamboa D. Multiple foraging strategies in a marine apex predator, the Galapagos sea lion Zalophus wollebaeki. Mar Ecol Prog Ser. 2008;363:299-309.

32. Villegas-Amtmann S, Jeglinski JW, Costa DP, Robinson PW, Trillmich F. Individual foraging strategies reveal niche overlap between endangered Galapagos pinnipeds. PLOS ONE. 2013;8(8):e70748.

33. Viviant M, Trites AW, Rosen DA, Monestiez P, Guinet C. Prey capture attempts can be detected in Steller sea lions and other marine predators using accelerometers. Polar Biol. 2010;33(5):713-9.

34. Viviant M, Monestiez P, Guinet C. Can we predict foraging success in a marine predator from dive patterns only? Validation with prey capture attempt data. PLoS ONE. 2014;9(3):e88503.

35. Volpov BL, Hoskins AJ, Battaile BC, Viviant M, Wheatley KE, Marshall G, Abernathy K, Arnould JP. Identification of prey captures in Australian fur seals (Arctocephalus pusillus doriferus) using head-mounted accelerometers: field validation with animal-borne video cameras. PLoS ONE. 2015;10(6):e0128789.

36. Volpov BL, Rosen DA, Hoskins AJ, Lourie HJ, Dorville N, Baylis AM, Wheatley KE, Marshall G, Abernathy K, Semmens J, Hindell MA. Dive characteristics can predict foraging success in Australian fur seals (Arctocephalus pusillus doriferus) as validated by animal-borne video. Biol Open. 2016:5(3):262-71.

37. Ware C, Trites AW, Rosen DA, Potvin J. Averaged propulsive body acceleration (APBA) can be calculated from biologging tags that incorporate gyroscopes and accelerometers to estimate swimming speed, hydrodynamic drag and energy expenditure for Steller sea lions. PLOS ONE. 2016;11(6):e0157326.

38. Wilson R, Steinfurth A, Ropert-Coudert Y, Kato A, Kurita M. Lip-reading in remote subjects: an attempt to quantify and separate ingestion, breathing and vocalisation in free-living animals using penguins as a model. Mar Biol. 2002;140(1):17-27.

39. Wisniewska DM, Johnson M, Teilmann J, Rojano-Doñate L, Shearer J, Sveegaard S, Miller LA, Siebert U, Madsen PT. Ultra-high foraging rates of harbor porpoises make them vulnerable to anthropogenic disturbance. Curr Biol. 2016;26(11):1441-6.

40. Ydesen KS, Wisniewska DM, Hansen JD, Beedholm K, Johnson M, Madsen PT. What a jerk: prey engulfment revealed by high-rate, supercranial accelerometry on a harbour seal (Phoca vitulina). J Exp Biol. 2014;217(13):2239-43.

\section{Publisher's Note}

Springer Nature remains neutral with regard to jurisdictional claims in published maps and institutional affiliations.

\footnotetext{
Ready to submit your research? Choose BMC and benefit from:

- fast, convenient online submission

- thorough peer review by experienced researchers in your field

- rapid publication on acceptance

- support for research data, including large and complex data types

- gold Open Access which fosters wider collaboration and increased citations

- maximum visibility for your research: over 100M website views per year
}

At $\mathrm{BMC}$, research is always in progress.

Learn more biomedcentral.com/submissions 